OPEN ACCESS

Edited by:

Francisco Lopez-Munoz, Universidad Camilo José Cela, Spain

Reviewed by: Ichiro Sakuma, Hokko Memorial Hospital, Japan Hugo Caire Castro-Faria-Neto, Fundação Oswaldo Cruz (Fiocruz),

Brazil

${ }^{*}$ Correspondence: Anding Xu tli@@inu.edu.cn

${ }^{\dagger}$ These authors have contributed equally to this work.

Specialty section: This article was submitted to Neuropharmacology, a section of the journal Frontiers in Neuroscience

Received: 26 January 2018 Accepted: 25 May 2018 Published: 19 June 2018

Citation: Zhang T, Lu D, Yang W, Shi C, Zang J, Shen L, Mai H and Xu A (2018) HMG-CoA Reductase Inhibitors Relieve Endoplasmic Reticulum Stress by Autophagy Inhibition in Rats With Permanent

Brain Ischemia.

Front. Neurosci. 12:405. doi: 10.3389/fnins.2018.00405

\section{HMG-CoA Reductase Inhibitors Relieve Endoplasmic Reticulum Stress by Autophagy Inhibition in Rats With Permanent Brain Ischemia}

\author{
Tao Zhang ${ }^{1 \dagger}$, Dan Lu ${ }^{2,3+}$, Wanyong Yang $2,3+$, Changzheng Shi4, Jiankun Zang ${ }^{2,3}$, \\ Lingling Shen ${ }^{2,3}$, Hongcheng Mai ${ }^{2,3}$ and Anding $\mathrm{Xu}^{2,3 *}$
}

1 Department of Cardiology, The First Affiliated Hospital of Jinan University, Guangzhou, China, ${ }^{2}$ Department of Neurology and Stroke Center, The First Affiliated Hospital of Jinan University, Guangzhou, China, ${ }^{3}$ Clinical Neuroscience Institute, Jinan University, Guangzhou, China, ${ }^{4}$ Department of Radiology, The First Affiliated Hospital of Jinan University, Guangzhou, China

Exploring and expanding the indications of common clinical drugs, such as statins, is important to improve the prognosis of patients with permanent cerebral infarction. It has been suggested that reversing the defects in cellular autophagy and ER stress with statin therapy may be a potential treatment option for reducing ischemic damage. Male Sprague-Dawley rats underwent permanent middle cerebral artery occlusion (PMCAO) by electrocoagulation surgery. Atorvastatin (ATV, $10 \mathrm{mg} / \mathrm{kg} / \mathrm{day}$ ) or vehicle was administered intraperitoneally. Rats were divided into the vehicletreated (SHAM), ATV pretreatment for MCAO (AMCAO), and 3-methyladenine (3MA) combined with ATV pretreatment (3MAMCAO) groups. Magnetic resonance imaging, as well as immunohistochemical and Western blot assessments, were performed $24 \mathrm{~h}$ after MCAO. Each ATV-treated group demonstrated significant reductions in infarct volume compared with that in the vehicle-treated group at $24 \mathrm{~h}$ after MCAO, which was associated with autophagy reduction and ER stress attenuation in neurons and neovascularization. Next, Western blotting was used to detect the levels of the autophagy-related proteins LC3B and P62 and of ER stress pathway proteins. However, 3MA significantly partially inhibited the ER stress pathway via limiting the autophagic flux in the AMCAO group. In conclusion, our results imply that the neuroprotective function of ATV depends on autophagic activity to diminish ER stress-related cell apoptosis in rats with $\mathrm{PMCAO}$ and suggest that compounds that inhibit autophagic activity might reduce the neuroprotective effect of ATV after brain ischemia.

Keywords: atorvastatin, 3-methyladenine, autophagy, endoplasmic reticulum stress, brain ischemia

\section{INTRODUCTION}

Stroke is a leading cause of death and disability worldwide according to reports by the World Heart Association, and ischemic stroke is the most common form, accounting for $87 \%$ of all strokes (Bin Abdulhak et al., 2014). Although great progress had been made in vascular recanalization, only $8.57 \sim 11.90 \%$ of patients were treated in the therapy time window $(\leq 3 \sim 4.5 \mathrm{~h}$ ) with intravenous (IV) recombinant tissue plasminogen activator (rt-PA) and $11.75 \%$ with endovascular treatment from 2010 to 2014 (Tekle et al., 2012; Zhang et al., 2016b,a). Achieving the proper balance of statin therapy will improve the prognosis of ischemic stroke patients. 
In recent years, atorvastatin (ATV), an important 3-hydroxy3-methylglutaryl-coenzyme A (HMG-CoA) reductase inhibitor (statin), was suggested to increase the odds of better primary outcomes compared to treatment without statins after thrombolysis (Cappellari et al., 2013, 2015). Additionally, some animal studies have reported the amelioration of ischemic brain damage via the downregulation of 12/15-lipoxygenase (LOX), p38 mitogen activated protein kinase (p38 MAPK) and cytosolic phospholipase A2 (cPLA2) expression (Cui et al., 2010) or the expression of high mobility group box-1 protein (HMGB1) and HMGB1 receptors [receptor for advanced glycation end products (RAGE) and toll-like receptor 4 (TLR4)] (Wang et al., 2010). However, additional work is necessary to improve the benefit of ATV in ischemic stroke patients. It has been suggested that reversing the defects in cellular autophagy and endoplasmic reticulum (ER) stress (Tripathi et al., 2016) with statin therapy may be a potential treatment option for reducing ischemic damage.

The ER plays an essential role in the lipid and protein biogenesis necessary for cellular homeostasis, and autophagy is induced by nutrient deprivation under ER stress conditions to protect cells against death. ER and autophagy are noteworthy for the link between intracellular metabolism and ischemia. A previous study demonstrated that the autophagy-related proteins microtubule-associated proteins light chain 3 (LC3B) and sequestosome 1 (SQSTM1, p62) were significantly upregulated in penumbra neurons after focal cerebral ischemia (Rami and Kogel, 2008), whether ischemia-induced autophagy was beneficial or detrimental (Kubota et al., 2010). ATV activated autophagy after ischemic stroke, inhibited apoptosis, and promoted recovery of neurological function. However, ATV was suggested to prevent apoptosis and attenuate nerve cell injury by acting through the protein kinase-like endoplasmic reticulum kinase (PERK) phosphorylation of the eukaryotic initiation factor 2 (eIF2a)/caspase-3 pathway (Yang and $\mathrm{Hu}, 2015$ ), but there was no detection of other key factors of the ER stress pathway. In a recent review, ER stress can induce autophagy processes mainly through the following signaling pathways: inositolrequiring enzyme 1 (IRE1- $\alpha$ )/phospho-c-Jun N-terminal kinases (JNKs)/X box-binding protein-1 (XBP1), PERK-eIF2 $\alpha$-activating transcription factor 4 (ATF4), and activating transcription factor 6 (ATF6) (Wang et al., 2018). Furthermore, whether ATV intervenes in the connection between autophagy and ER stress and how to improve the protective effect by combination with other treatments remain to be studied.

Therefore, in this present study, we focused on the protective effects of ATV pretreatment on neurons, endothelium, astrocytes and microglia after brain ischemia through autophagic activity and ER stress and proposed the interplay between ER stress and autophagy in this situation.

\section{MATERIALS AND METHODS}

\section{Animal Model}

All animal procedures were conducted in strict accordance with the recommendations of the National Institutes of Health
Guidelines (NIH Publications No. 8023, revised 1978) for the Care and Use of Laboratory Animals. The experimental protocols were approved by Competent Ethics Committees of Jinan University. A total of 40 male Sprague-Dawley rats (250-300 g) were purchased from the Animal Experiment Center of Southern Medical University, Guangzhou, China. Twenty rats were subjected to middle cerebral artery occlusion (MCAO) and 8 rats served as sham-operated (SHAM) controls. Permanent MCAO (PMCAO) was performed by electrocoagulation as previously described (Zhu et al., 2016). Briefly, the rats were anesthetized with chloral hydrate $(0.3 \mathrm{mg} / \mathrm{kg})$ by intraperitoneal injection. Under an operating microscope, the left MCA was exposed through a burr hole and occluded by electrocoagulation. The SHAM animals underwent the same surgical procedures except for the electrocoagulation of the middle cerebral artery. The body temperature was maintained at $37 \pm 0.5^{\circ} \mathrm{C}$ during the surgery. At 3 days before MCAO, rats in the ATV group (AMCAO) and vehicle group (each group $N=8$ ) were intraperitoneally injected with $10 \mathrm{mg} / \mathrm{kg}$ of ATV (Sigma-Aldrich, Fluka, St. Louis, MO, United States) and isopycnic vehicle, respectively, and then continuously injected once a day for 3 days. 3-Methyladenine (3MA; Sigma-Aldrich, Fluka, St. Louis, MO, United States; $5 \mathrm{mmol} / \mathrm{L}$ ) at a dosage of $4 \mathrm{~mL} / \mathrm{kg} /$ day (3MAMCAO group, $N=4$ ) and isopycnic vehicle were given by tail vein injection (Zhao et al., 2016) (dissolved in $1 \mathrm{~mL}$ of saline) $1 \mathrm{~h}$ before the intraperitoneal injection of ATV and vehicle every time according to the previous studies to inhibit autophagy in the 3MAMCAO group (Guo et al., 2017). Only one animal was excluded because of model failure in the 3MAMCAO group. All animals survived for $24 \mathrm{~h}$ after MCAO before the following experiments.

\section{Magnetic Resonance Imaging (MRI)}

The MRI was conducted by a Discovery $7503.0 \mathrm{~T}$ scanner with an 8-channel wrist coil (GE Healthcare, Milwaukee, WI, United States) before heart perfusion, and the other 3 rats in each group were used for Western blotting. Five rats in each group were randomly anesthetized with $10 \%$ chloral hydrate and placed in a prone position before scanning. All experimental animals underwent coronal T1-weighted imaging, T2-weighted imaging (T2WI), diffusion-weighted imaging (DWI), and T2*-weighted imaging, with scan parameters as follows. A coronal T2 sequence [effective echo time (TE): $81 \mathrm{~ms}$, TR: $4600 \mathrm{~ms}$; plane resolution: $0.25 \mathrm{~mm} \times 0.27 \mathrm{~mm}$; 17 slices, $2.0 \mathrm{~mm}$ in thickness] was used for T2-derived infarct measurement. DWI scans using singleshot echo planar imaging (EPI) pulse sequence were applied. Imaging parameters for DWI acquisition included field of view $(\mathrm{FOV})=80 \mathrm{~mm} \times 50 \mathrm{~mm}$, slice thickness $/$ spacing $=2 / 0 \mathrm{~mm}$, repetition time $(\mathrm{TR}) / \mathrm{TE}=3000 / 70 \mathrm{~ms}$, matrix $=96 \times 128$, number of excitations $(\mathrm{NEX})=4, b$-values $=800 \mathrm{~s} / \mathrm{mm}^{2}$, total scanning time $=42 \mathrm{~s}$. T2 $*$-weighted signal change was assessed by the parameters TE: $4,8,12,17,21,25,29,33,37,42,46,50,54,58$, 63 , and $67 \mathrm{~ms}$, TR: $126 \mathrm{~ms}$, matrix $160 \times 160$, FOV $80 \times 80 \mathrm{~mm}$, and 17 contiguous slices of $2.0 \mathrm{~mm}$ in thickness.

\section{NissI Staining}

Animals were perfused with $0.9 \%$ saline followed by $4 \%$ paraformaldehyde and $0.1 \%$ glutaraldehyde in $0.1 \mathrm{M}$ phosphate 

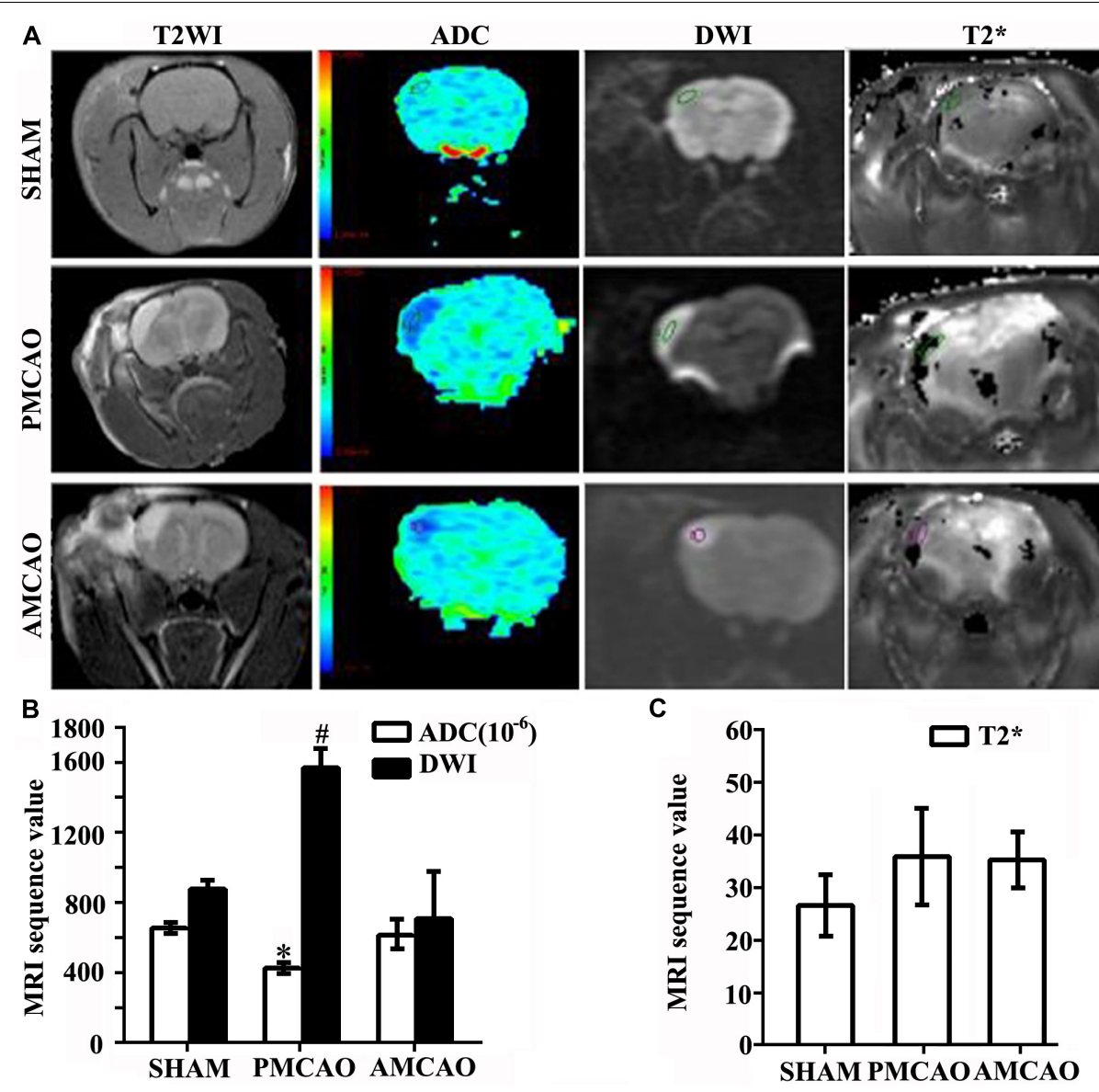

FIGURE 1 | Infarct volumes. (A) MRI images showing changes in the ischemia area. Areas in pale blue in the ADC images represent the signal of the free diffusion of extracellular free water molecules; areas in dark blue reflect the limited diffusion of extracellular free water molecules. (B) Histogram showing changes in the values of the ADC and DWI in all groups. Quantitative analysis demonstrating the ADC values in the PMCAO group vs. the SHAM group (* $p<0.05)$, the AMCAO group vs. the PMCAO group ( $* p<0.05)$, and the DWI values in the SHAM and AMCAO groups vs. the PMCAO group $\left({ }^{\#} p<0.01\right)$. Mean \pm SEM, $N=5$. (C) Histogram showing changes in the values of T2* in all groups. Mean $\pm \mathrm{SEM}, N=5$.

buffer ( $\mathrm{pH}$ 7.4). Brains were harvested and postfixed overnight at $4^{\circ} \mathrm{C}$. Coronal sections $(10 \mu \mathrm{m})$ were cut using a vibratome and underwent Nissl staining according to previous methods. Briefly, brain sections $(10 \mu \mathrm{m})$ were stained with Cresyl violet solution (Beyotime, Shanghai, China) at $50^{\circ} \mathrm{C}$ for $45 \mathrm{~min}$. After dehydration in serially diluted ethanol and clearing in xylene, images of the Nissl-stained sections were captured using a microscope (Leica, Germany), and quantitative analysis was performed using ImageJ software (National Institutes of Health, United States).

\section{Immunofluorescence}

Immunofluorescent staining was performed as previously described (Garbuzova-Davis et al., 2014). Similar to the Nissl staining, before immunofluorescence, the animals were perfused with $0.9 \%$ saline followed by $4 \%$ paraformaldehyde and $0.1 \%$ glutaraldehyde in $0.1 \mathrm{M}$ phosphate buffer $(\mathrm{pH}$ 7.4). Brains were harvested and postfixed overnight at $4^{\circ} \mathrm{C}$. Coronal sections $(10 \mu \mathrm{m})$ were cut using a vibratome. The sections were incubated with primary antibodies [mouse anti-P62 monoclonal antibody, 1:250, catalog number (cat\#) MABC32, Merck Millipore, Hong Kong, China; rabbit anti-LC3B polyclonal antibody, 1:200, cat\# 2775, Cell Signaling Technology (CST), Danvers, MA, United States; rabbit anti-CD34 monoclonal antibody, as a type I transmembrane glycophosphoprotein expressed by vascular endothelium, 1:100, cat\# ab8536, Abcam, Shanghai, China; mouse anti-glial fibrillary acidic protein (GFAP) monoclonal antibody, cat\# ab4648, Abcam; mouse anti- ionized calcium-binding adapter molecule 1 (Iba-1), as a marker of microglia, monoclonal antibody, cat\# sc32725, Santa Cruz, Shanghai, China] at $4^{\circ} \mathrm{C}$ overnight, washed with 0.01 PBS for $3 \mathrm{~min} \times 5 \mathrm{~min}$, and then incubated with secondary antibodies (488 nm FITC-labeled goat anti-rabbit IgG, $546 \mathrm{~nm}$ TRITC-labeled goat anti-mouse IgG, 1:1000 and $305 \mathrm{~nm}$ labeled goat anti-mouse IgG; Yeasen, Shanghai, China) for $2 \mathrm{~h}$ at room temperature. After immunostaining, the sections were counterstained with 4',6-diamidino-2-phenylindole (DAPI, 1:10000, Beyotime Biotechnology, Shanghai, China) to detect cell nuclei, and the slices were photographed under a fluorescence microscope (Leica DM1000, Germany). 


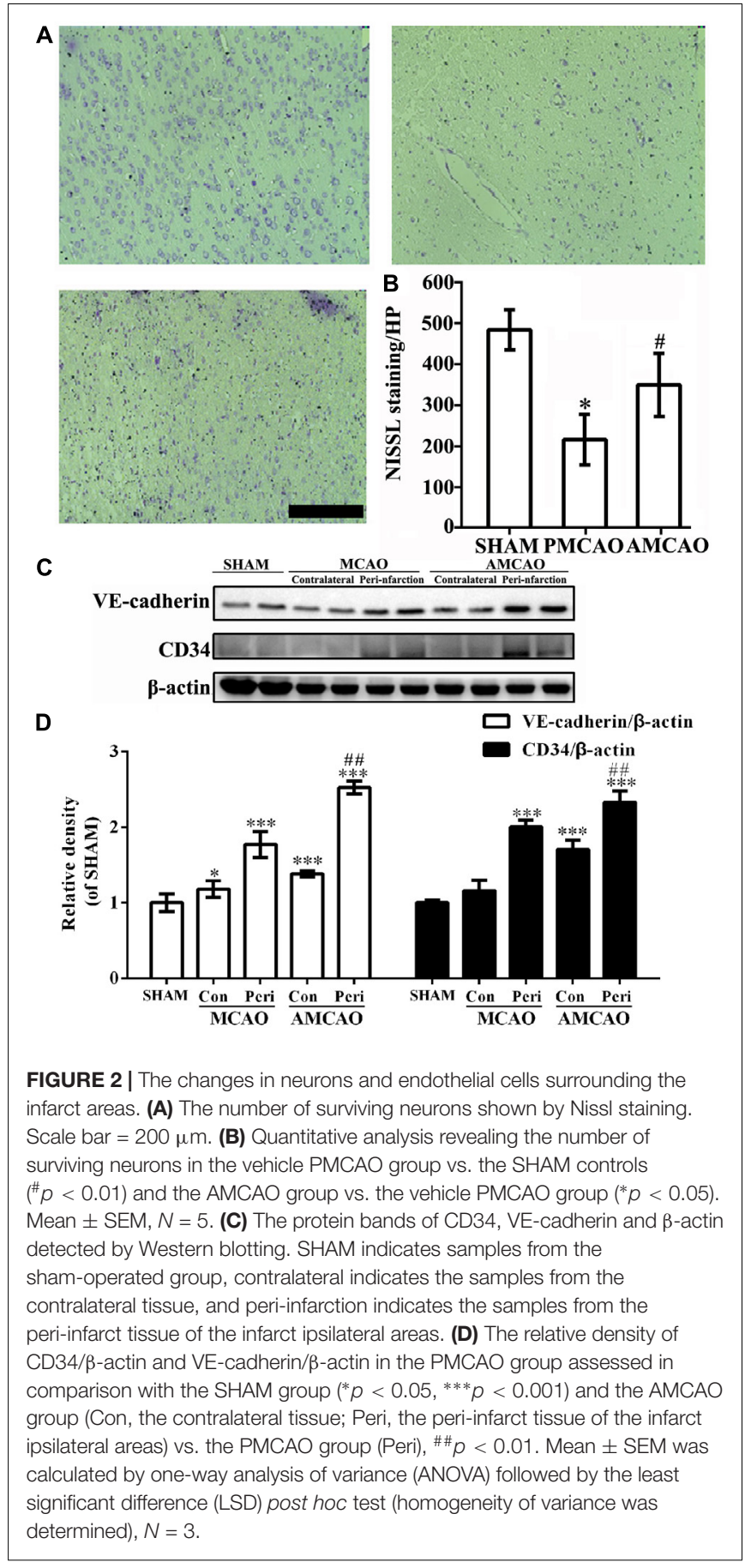

\section{Transmission Electron Microscopy (TEM)}

Fresh peri-infarct brain tissue samples (approximately $1 \mathrm{~mm}^{3}$ ) were fixed in $2.5 \%$ glutaraldehyde at $4^{\circ} \mathrm{C}$ overnight when the brain tissues were collected for Western blotting. Ultrathin sections were cut from the fixed blocks, dehydrated, embedded in epoxy resin, cut off, which is a standard process, and finally examined using an electron microscope (JEM-1200, JEOL Ltd., Tokyo, Japan) at $80 \mathrm{kV}$. Moreover, three areas in the ipsilateral peri-infarct cortex in each section were chosen.

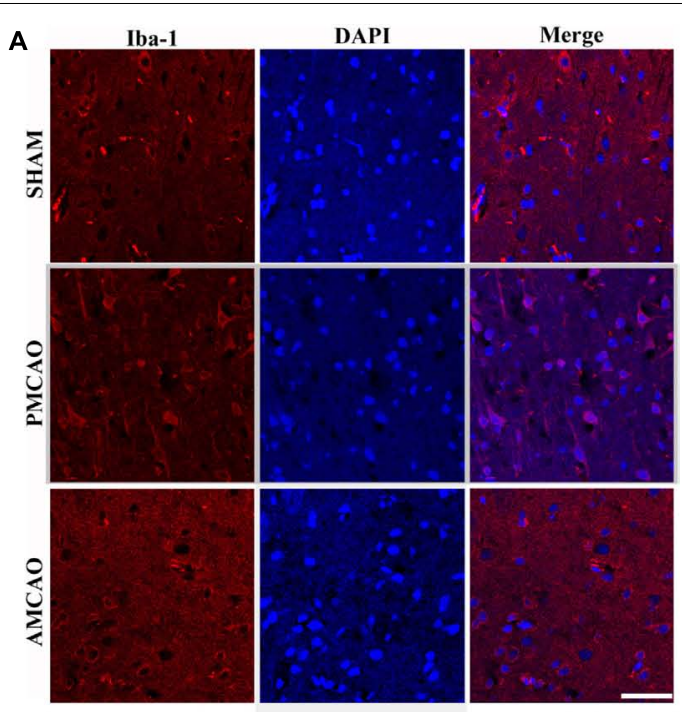

B

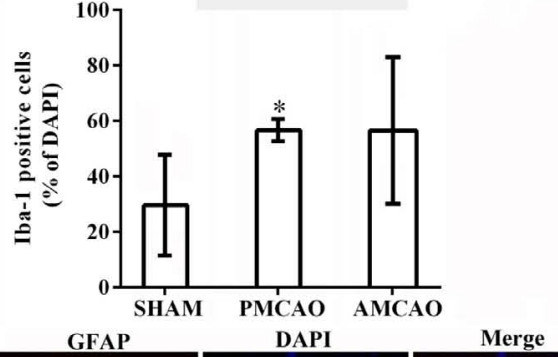

C
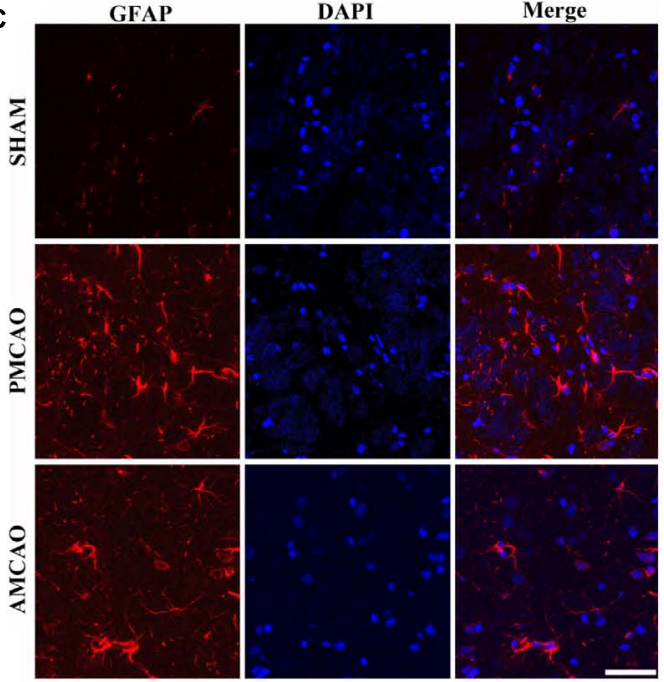

D

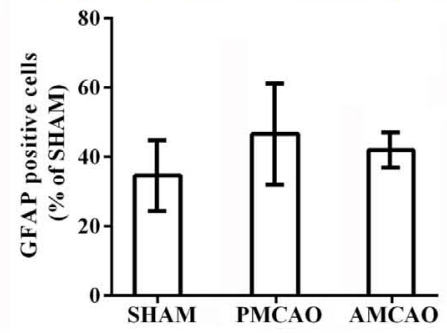

FIGURE 3 | The changes in microglia and astrocytes surrounding the infarct areas. (A) Immunofluorescence micrographs showing differences in the number of Iba-1-positive cells (red) surrounding the infarct area in all

(Continued) 
FIGURE 3 | Continued

experimental groups. Scale bar $=50 \mu \mathrm{m}$. (B) The lba-1-labeled particle numbers (red)/DAPI (blue) in the PMCAO group vs. the SHAM group $\left({ }^{*} p<0.05\right)$. (C) Immunofluorescence micrographs showing differences in the number of GFAP-positive cells (red) surrounding the infarct area in all experimental groups. Scale bar $=50 \mu \mathrm{m}$. (D) The GFAP-labeled particle numbers (red)/DAPI (blue) detected in the SHAM, vehicle PMCAO, and AMCAO groups. Mean $\pm \mathrm{SEM}, N=5$.

\section{Western Blotting}

Western blotting was performed as previously described ( $\mathrm{Lu}$ et al., 2015). Fresh peri-infarct brain tissues were lysed with cell lysis buffer containing a protease inhibitor (Beyotime, Shanghai, China). The protein concentration was determined using a bicinchoninic acid protein assay kit (Beyotime, Shanghai, China). Samples containing equal amounts of protein $(30 \mu \mathrm{g})$ were separated via sodium dodecyl sulfate-polyacrylamide gel electrophoresis and transferred to polyvinylidene difluoride membranes for blotting with antibodies purchased from CST, Danvers, MA, United States $\beta$-actin, 1:1000, cat $\#$ 4970; pPERK, 1:1000, cat\# 3179; the phospho-eIF2 $\alpha$ (peIF2 $\alpha$ ), 1:1000, cat\# 5324; ATF4, which functions in the PERK and eIF2 $\alpha$ ER stress responsive pathway, 1:1000, cat\# 11815; ATF6, which is a transcription factor that activates target genes for the unfolded protein response (UPR) during ER stress, 1:1000, cat\# 65880; the phosphorylation of the transcription factors of the nuclear factor кB (pNF-кB), 1:1000, cat\# 3033; phospho-p38 MAPK (pP38), 1:1000, cat\# 4511; pJNK, 1:1000, cat\# 4668; C/EBP-homologous protein (CHOP), which participates in programmed cell death of ER-stressed cells by promoting protein synthesis and oxidative stress inside the ER, 1:1000, cat\# 2895; Bcl-2, 1:1000, cat\# 3498; calnexin, which is a chaperone characterized by assisting protein folding and quality control to ensure that only properly folded and assembled proteins proceed further along the secretory pathway, 1:1000, cat\# 2679; Bip, 1:1000, cat\# 3177; the ERresiding protein endoplasmic oxidoreductin-1 (Ero1-L $\alpha$ ), which is an ER membrane-associated $\mathrm{N}$-glycoprotein that promotes oxidative protein folding, 1:1000, cat\# 3264; the nuclear factorlike 2 (Nrf2), which maintains cellular homeostasis through the regulation of basal levels of antioxidant response genes, 1:1000, cat\# 12721; cleaved-caspase 3, which is a critical executioner of apoptosis, 1:1000, cat\# 9664; hypoxia-inducible factor 1 (HIF1$\alpha$ ), which plays a critical role in the cellular response to hypoxia, 1:1000, cat\# ab51608 from CST; phospho-mammalian target of rapamycin (pmTOR), which has been shown to be activated by rapamycin to prevent ER stress and induce autophagy (Tripathi et al., 2016)). Additional antibodies, including IRE1- $\alpha$, which is proposed to be a proximal sensor for the UPR that transmits the unfolded protein signal across the ER membrane; phosphoIRE1- $\alpha$ (1:1000; cat\# ab48187); and vascular endothelial cadherin (VE-cadherin), which is localized at the intercellular junctions of endothelial cells where it is thought to play a role in the cohesion and organization of intercellular junctions (1:200, cat\# WL02033), were purchased from Abcam (Wanleibio, Shenyang, China). The relevant horseradish peroxidase (HRP)-conjugated secondary antibodies (Yeasen, Shanghai, China) were incubated for $1 \mathrm{~h}$. The SuperSignal substrate (Wanleibio, Changchun, China) was used to visualize the immunoreactive proteins. A Western Imaging System (Tanon 5200, Shanghai, China) and Quantity One software were used to measure the protein density. The density of protein bands was exposed and analyzed with a Tanon 2500 Gel Imaging System (Tanon, Shanghai, China). The raw data was submitted in the Supplementary Data Sheet S1.

\section{TTC (2, 3,5-Triphenyltetrazolium Chloride) Staining}

Twenty-four hours after MCAO, 4 rats in each of the PMCAO, AMCAO, and 3MAMCAO groups were sacrificed. The brains were cut into 3-mm coronal slices, placed in 2\% TTC (Sangon Biotech, Shanghai, China) solution, and incubated at $37^{\circ} \mathrm{C}$ for $5 \mathrm{~min}$ in the dark. Healthy tissues in the TTC solution were dyed red, and infarct tissues were white. The stained sections were captured by a camera, the images were analyzed using ImageJ software and then, the percentage of infarct volume was calculated (Zhang et al., 2011).

\section{Statistical Analysis}

The data are presented as the mean \pm standard error of the mean (SEM). One-way analysis of variance (ANOVA) was used to compare the average values between the different groups and was followed by an appropriate least significant difference (LSD) post hoc test (if homogeneity of variance was determined) or Tamhane's T2 post hoc test (if homogeneity of variance was not determined) using the SPSS statistical software package (version 13.0; SPSS, Inc., Chicago, IL, United States). $p<0.05$ was considered to indicate a statistically significant difference.

\section{RESULTS}

\section{The Changes in Infarct Volume and Cells Surrounding Infarct Areas}

Twenty-four hours after MCAO $(N=5)$, significant changes in signal intensity were observed in the ipsilateral hemisphere, as evaluated by DWI and the apparent diffusion coefficient (ADC). Compared to the matched area in the SHAM controls, the cerebral infarct area in the PMCAO animals revealed higher signals on T2-weighted imaging and on DWI and dark blue signals on the ADC maps, which were reversed to nearly normal signals by ATV pretreatment (Figure 1A). Quantitative analysis demonstrated that the DWI values were lower in the SHAM $(878 \pm 44.9)$ and AMCAO $(790 \pm 184)$ groups than in the PMCAO group $(1575 \pm 100.3, p<0.01)$. The ADC value in the PMCAO group $(426 \pm 22.4)$ was lower than that in the SHAM group $(653 \pm 28.0, p<0.05)$, and the ADC value was higher in the AMCAO group $(621 \pm 80.6)$ than in the PMCAO group $(p<0.05$, Figure 1B). Moreover, there was no significant difference in the blood leakage levels obtained at $24 \mathrm{~h}$ post-MCAO by $\mathrm{T}^{*}$-weighted imaging among the three groups (Figure 1C).

Next, the number of intact cells in the injured brain areas at $24 \mathrm{~h}$ after PMCAO injury was significantly reduced 

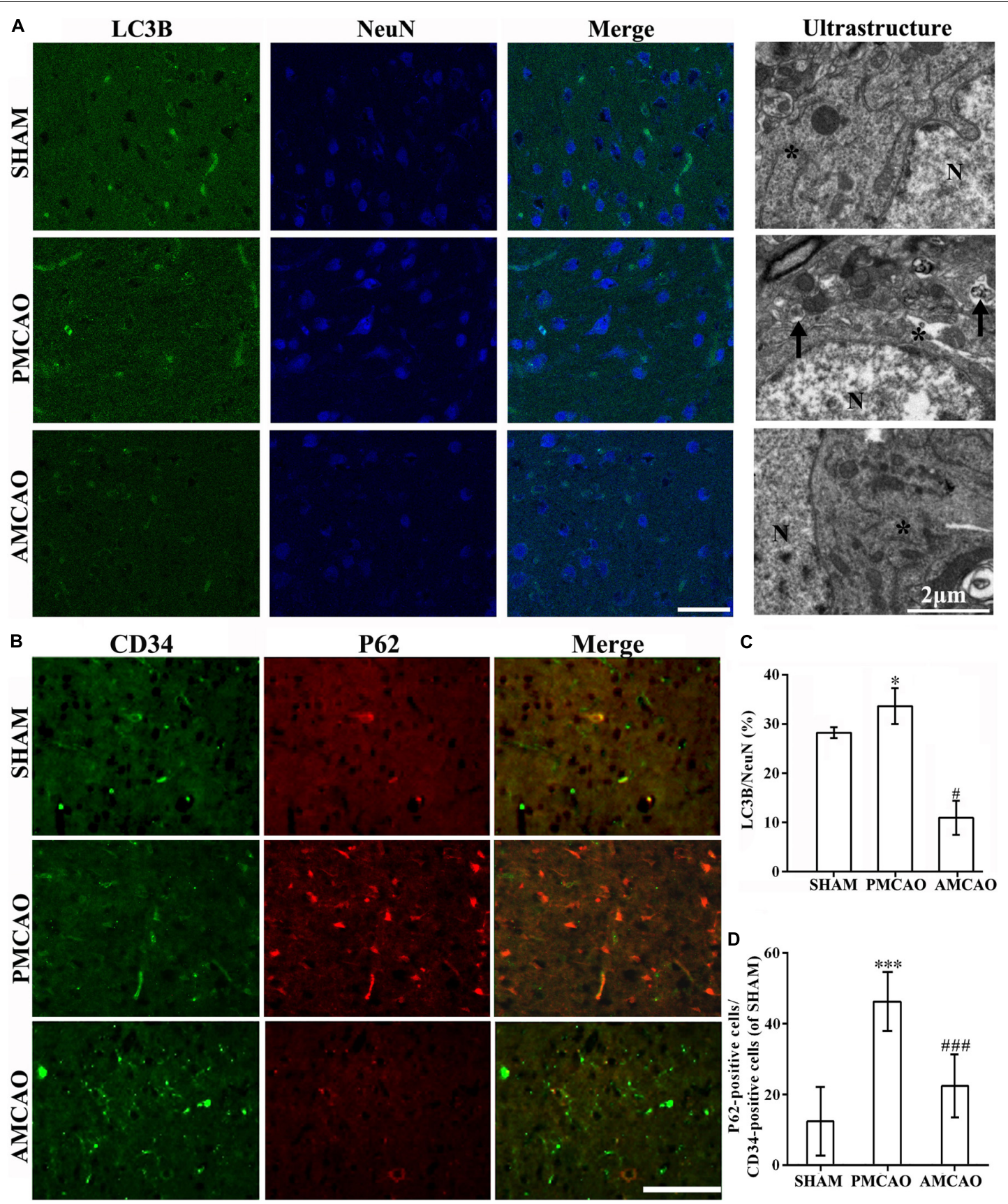

FIGURE 4 | Protection of ATV pretreatment in vascular endothelial cells and neurons against ischemic brain injury by suppressing autophagic activity.

(A) Immunofluorescence micrographs showing differences in the number of LC3B (red) and NeuN-positive cells (blue) in the ipsilateral injury area in all experimental groups. Scale bar $=50 \mu \mathrm{m}$. The ultrastructural results of each group shown by electron microscopy in the right column. The star, $\mathrm{N}$ and arrow, respectively, indicate the ribosomes and surrounding ribosomes, nucleus and autophagosomes. Scale bar $=2 \mu \mathrm{m}$. (B) Immunofluorescence micrographs showing the differences in the numbers of CD34- (green) and P62-positive cells (red) in the ipsilateral injury area in all experimental groups. Scale bar $=100 \mu \mathrm{m}$. (C) The LC3B-labeled particle numbers/NeuN detected in the PMCAO group vs. the SHAM group $\left({ }^{*} p<0.05\right)$ and the AMCAO group $\left({ }^{\#} p<0.05\right)$. Mean \pm SEM, as calculated by one-way analysis of variance (ANOVA) followed by the least significant difference (LSD) post hoc test (homogeneity of variance was determined), $N=5$. (D) The P62-labeled particle numbers/CD34-positive cells detected in the PMCAO group vs. the SHAM group (*** $p<0.001$ ) and AMCAO group (\#\#\# $<0.001)$. Mean \pm SEM was calculated by one-way ANOVA followed by LSD post hoc test (homogeneity of variance was determined), $N=5$.

in the MCAO-injured animals treated with normal saline $\left(252 \pm 68.7 / \mathrm{mm}^{2}\right)$ compared with that in the SHAM controls $\left(564 \pm 53.6 / \mathrm{mm}^{2}\right)$, as detected by Nissl staining $(p<0.01)$, and showed shrunken cell bodies and condensed nuclei. However, the cell loss was significantly mitigated in the MCAO-injured animals treated with ATV $\left(407 \pm 86.1 / \mathrm{mm}^{2}\right)$ 

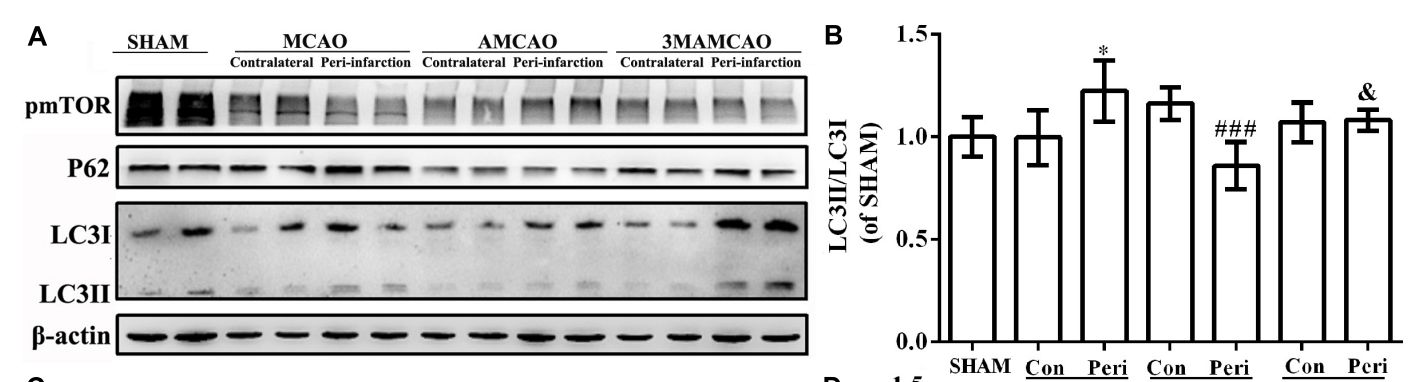

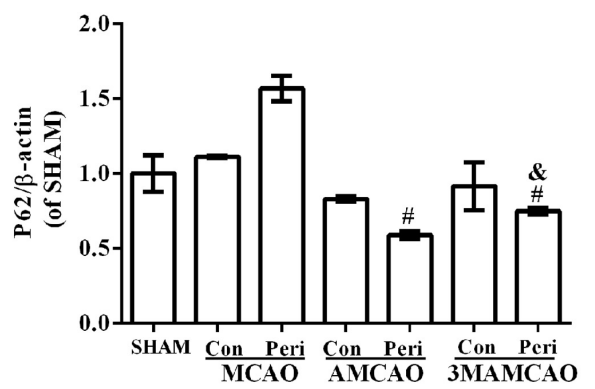

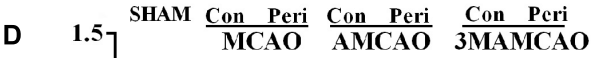

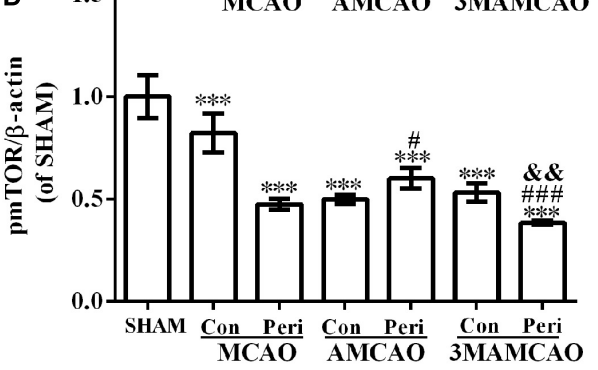

E

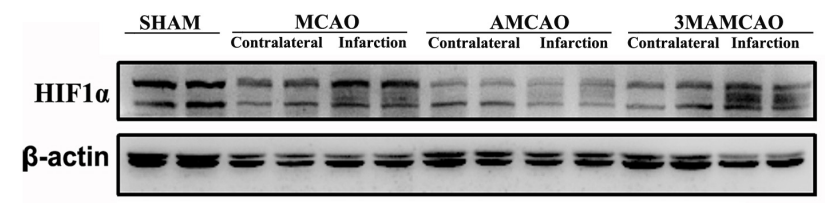

F

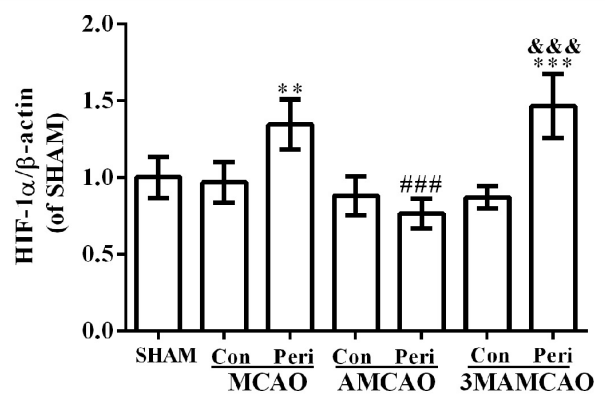

FIGURE 5 | The protein levels of pmTOR, LC3B, P62, and HIF1 $\alpha$. (A) The protein bands of pmTOR, LC3B, P62, and $\beta$-actin detected using Western blotting and exposed by Tanon 2500/2500R. (B) The relative densities of LC3II/LC3I, (C) P62/ $\beta$-actin, and (D) pmTOR/ $\beta$-actin in the PMCAO group assessed in comparison with the SHAM group ( $\left.{ }^{*} p<0.05,{ }^{* * *} p<0.001\right)$, the AMCAO group vs. the PMCAO group ( $p<0.05$, \#\# $\left.p<0.001\right)$, and in the AMCAO group vs. the 3MAMCAO group ( $\left.{ }^{\&} p<0.05,{ }^{\&} \& p<0.01\right)$. The mean \pm SEM of LC3II/LC3I and pmTOR/ $\beta$-actin was calculated by one-way analysis of variance (ANOVA) followed by the least significant difference (LSD) post hoc test (homogeneity of variance was determined), while the mean \pm SEM of P62/ $\beta$-actin was calculated by one-way ANOVA followed by Tamhane's T2 test (homogeneity of variance was not determined), $N=3$. (E) The protein bands of HIF1 $\alpha$ and $\beta$-actin detected using Western blotting and exposed by Tanon 2500/2500R. (F) The relative density of HIF1 $\alpha / \beta$-actin in the PMCAO group assessed in comparison with the SHAM group (** $p<0.01$, ${ }^{* * *} p<0.01$ ), the AMCAO group vs. the PMCAO group (\#\# $p<0.001$ ) and the AMCAO group vs. the 3MAMCAO group ( $\left.\& \& \& p<0.001\right)$. The mean \pm SEM of $\mathrm{HIF} 1 \alpha / \beta$-actin was calculated by one-way ANOVA followed by the LSD post hoc test (homogeneity of variance was determined), $N=3$.

compared with that in the vehicle PMCAO group $(p<0.05$, Figures 2A,B).

Additionally, the expression of CD34 and VE-cadherin proteins in the penumbra core of the MCAO group was significantly increased compared with that in the SHAM control group $\left({ }^{* *} p<0.001, N=3\right.$ ), while pretreatment with ATV significantly increased the levels of the CD34 and VE-cadherin proteins in the AMCAO animals compared to the vehicle-treated PMCAO animals ( ${ }^{\#} p<0.01, N=3$, Figures 2C,D).

The quantitative analysis revealed that the percentage of the microglia marker Iba-1-positive cells (Figure 3A) was dramatically increased to $56.66 \pm 3.97 \%$ in the peri-infarct area of the PMCAO-injured animals treated with normal saline compared with the percentage of $29.64 \pm 18.21 \%$ in the SHAM control animals $(p<0.05$, Figure 3B). There was no significant difference between the PMCAO-injured animals treated with ATV and the vehicle-treated PMCAO animals (Figures 3A,B). The analysis also revealed that the number of the astrocyte marker GFAP-positive cells (Figure 3C) was dramatically increased to $46.64 \pm 14.60 \%$ in the peri-infarct area of the PMCAO-injured animals treated with normal saline compared with $34.62 \pm 10.16 \%$ in the SHAM animals $(p<0.05$, 

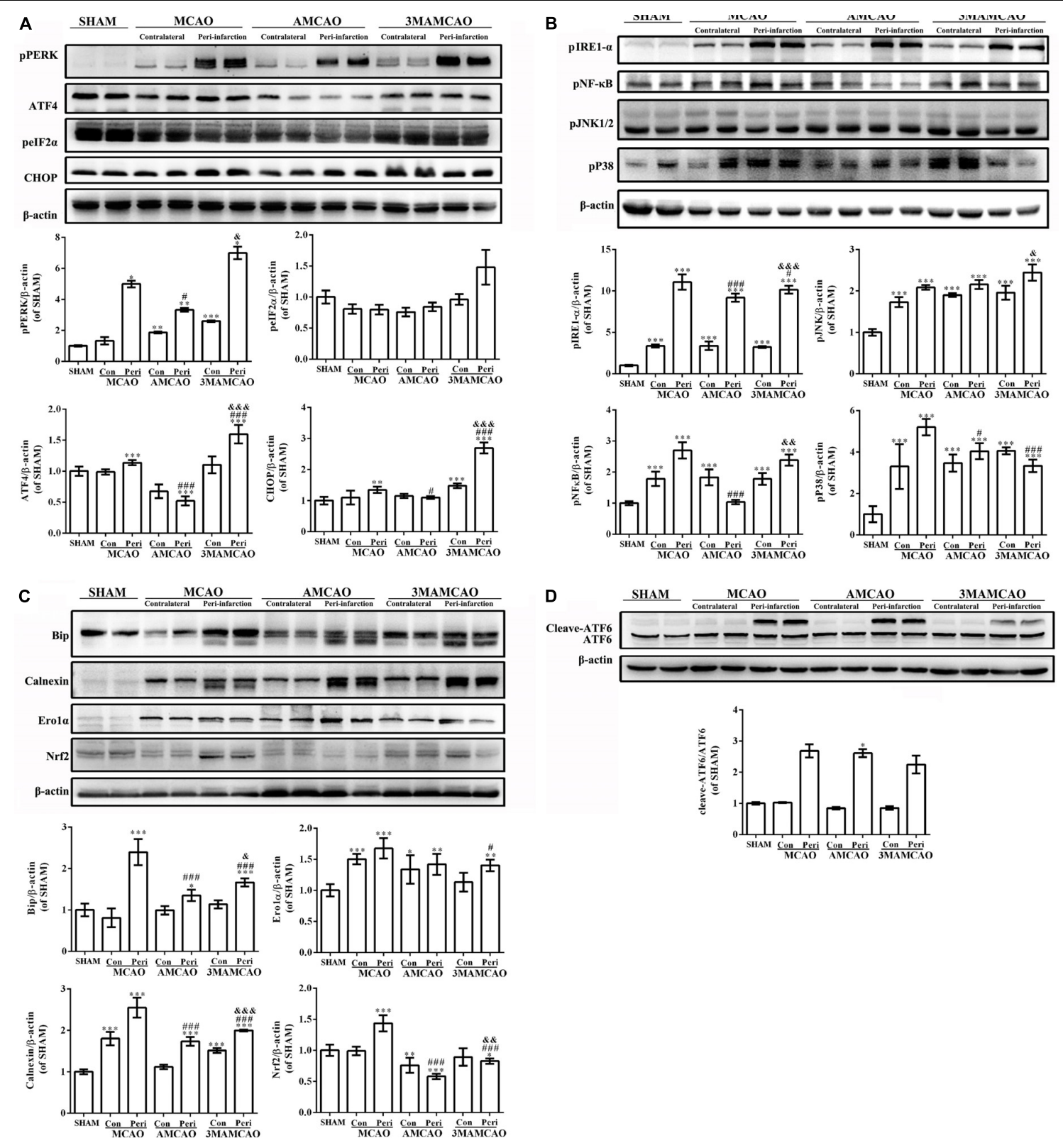

FIGURE 6 | Regulation of protein expression in the ER stress pathway by ATV and 3MA in ischemic stroke rats. (A) The protein bands of pPERK, pelF2 $\alpha$, ATF4, $\mathrm{CHOP}$, and $\beta$-actin detected by Western blotting and exposed by Tanon 2500/2500R. (B) The protein bands of ATF6 and $\beta$-actin detected by Western blotting and exposed by Tanon 2500/2500R. (C) The protein bands of pIRE-1 $\alpha$, pNF-кB, pP38, and pJNK detected using Western blotting and exposed by Tanon 2500/2500R. (D) The protein bands of Bip, calnexin, Ero1-L $\alpha$ and Nrf2 detected using Western blotting and exposed by Tanon 2500/2500R. The relative densities of the other proteins were assessed by the ratio of each ER stress protein to $\beta$-actin in comparison with the SHAM group $\left({ }^{*} p<0.05\right.$, $\left.{ }^{* *} p<0.01,{ }^{* * *} p<0.001\right)$, the AMCAO

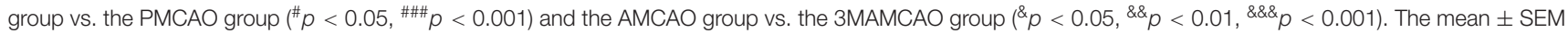
of pPERK/ $\beta$-actin, pelF2 $\alpha / \beta$-actin, and cleaved-ATF6/ATF6 was calculated by one-way analysis of variance (ANOVA) followed by the Tamhane's T2 test (homogeneity of variance was not determined), whereas the mean $\pm \mathrm{SEM}$ of $\mathrm{ATF} 4 / \beta$-actin, $\mathrm{CHOP} / \beta$-actin, $\mathrm{pIRE}-1 \alpha / \beta$-actin, $\mathrm{pNF}$ - $\mathrm{BB} / \beta$-actin, $\mathrm{pP} 38 / \beta$-actin, pJNK/ $\beta$-actin, Bip/ $\beta$-actin, calnexin/ $\beta$-actin, Ero1-L $\alpha / \beta$-actin, and Nrf2/ $\beta$-actin was calculated by one-way ANOVA followed by the least significant difference (LSD) post hoc test (homogeneity of variance was determined), $N=3$.

Figure 3D). There was no significant difference between the PMCAO-injured group treated with ATV and the vehicle PMCAO group (Figures 3C,D).

These findings were further confirmed in the animal experiment showing that pretreatment with ATV increased the expression of CD34 and VE-cadherin and the number of neurons in the PMCAO rats, and there was no significant difference in the number of glia cells between the PMCAOinjured group treated with ATV and the vehicle PMCAO group. 


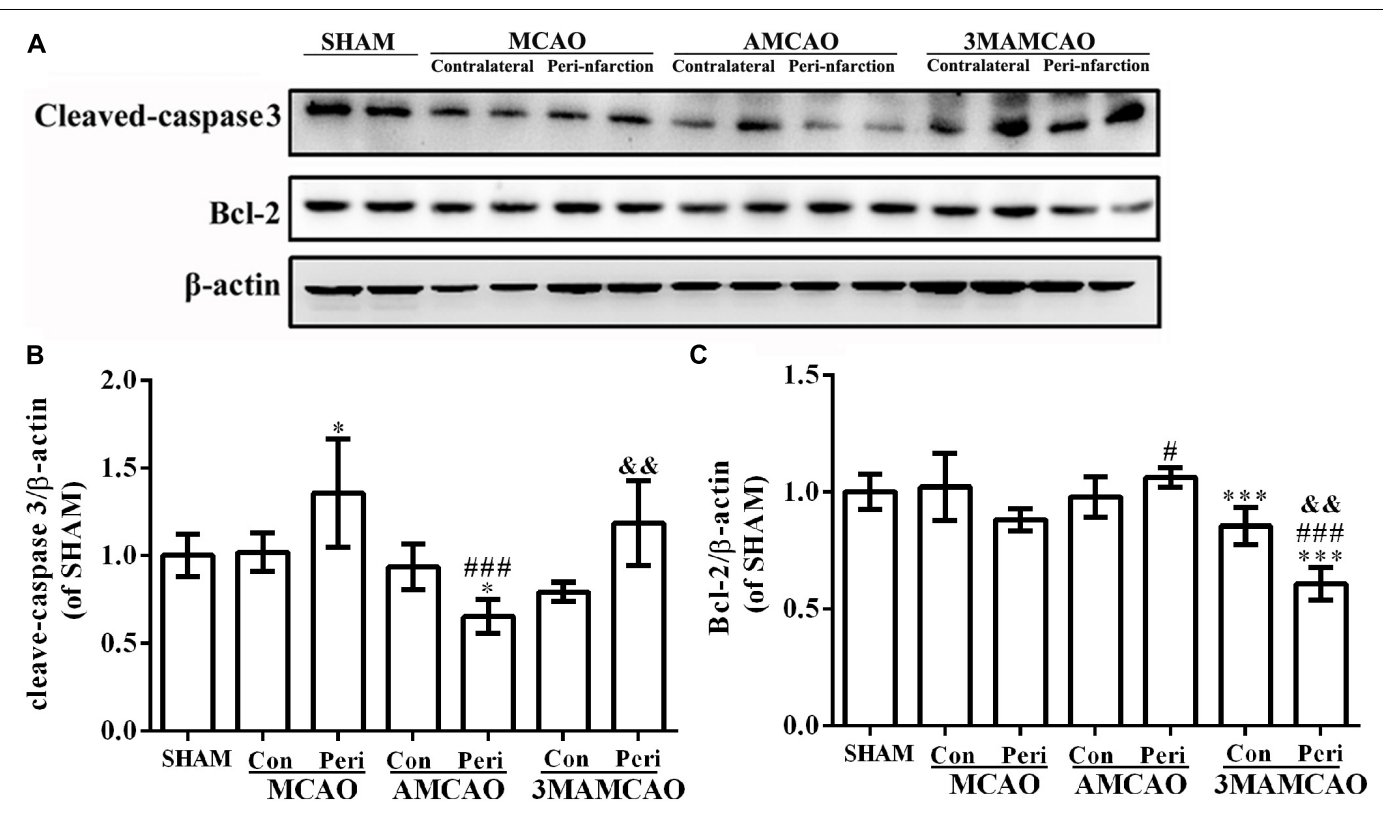

FIGURE 7 | Regulation of protein levels of cleaved-caspase 3 and Bcl-2 by ATV and 3MA in ischemic stroke rats. (A) The protein bands of cleaved-caspase 3 , Bcl-2 and $\beta$-actin detected by Western blotting. (B) The relative density of cleaved-caspase $3 / \beta$-actin in the PMCAO group assessed in comparison with the SHAM group $\left({ }^{*} p<0.05\right)$, and the AMCAO group vs. the PMCAO group (\#\#\# $\left.p<0.001\right)$ and the AMCAO group vs. the 3MAMCAO group (\&\& $\left.p<0.01\right)$. The mean \pm SEM of cleaved-caspase $3 / \beta$-actin was calculated by one-way analysis of variance (ANOVA) followed by the least significant difference (LSD) post hoc test (homogeneity of variance was determined), $N=3$. (C) The relative density of $\mathrm{Bcl}-2 / \beta$-actin in the PMCAO group assessed in comparison with the SHAM group (*** $p<0.001$ ) and the AMCAO group vs. the PMCAO group ( ${ }^{\prime} p<0.05$, \#\#\# $\left.<0.001\right)$ and the AMCAO group vs. the 3MAMCAO group ( \& $\left.p<0.01\right)$. The mean \pm SEM of Bcl-2/ $\beta$-actin was calculated by one-way ANOVA followed by the LSD post hoc test (homogeneity of variance was determined), $N=3$.

\section{Atorvastatin Promoted the Autophagic Activity of Vascular Endotheliocytes and Neurons}

The numbers of LC3-positive cells (Figure 4A) and P62-positive endothelial cells (Figure 4B) in the brain sections were analyzed with the imaging software ImageJ. The number of LC3-positive cells in the peri-infarct areas in the ATV-pretreated group was also significantly less than that in the vehicle PMCAO group $\left({ }^{\#} p<0.05\right.$, Figure 4C). Then, TEM was used to examine the morphological changes in neurons. Cortical neurons from the SHAM control rats contained normal-looking nuclei with abundant ER and relatively healthy-looking mitochondria and lysosomes. In contrast, cortical neurons surrounding the infarct areas subjected to PMCAO injury displayed an increase in the number of autophagosomes, which were identified as bubble-like vacuoles enclosing recognizable cytoplasmic structures. In the AMCAO group, the appearance of neurons and their organelles were less damaged after pretreatment with ATV, and fewer autophagosomes were generated from the ER in the vehicle PMCAO group (Figure 4A, Ultrastructure). The endothelium cells were labeled with the CD34 antibody, and the results showed that the numbers of P62-positive endothelial cells in the SHAM controls $\left({ }^{* *} p<0.001\right)$ and the peri-infarct areas of the ATVpretreated groups ( $\left.{ }^{\# \# \#} p<0.001\right)$ were significantly decreased compared with that in the vehicle PMCAO group (Figures 4B,D), suggesting that autophagy flux was blocked due to the failure of autophagosome degradation secondary to the P62 transition.
Activation of autophagy was further examined by Western blotting of LC3 and P62 proteins, two regulators in the autophagic cascade (Figure 5A). The results showed that the LC3II/LC3I ratio was increased in the vehicle PMCAO group ( ${ }^{*} p<0.05$ vs. the SHAM controls, LSD test), and the trend of P62 was increased from the baseline level (Tamhane's T2 test). The LC3II/LC3I conversion (Figure 5B, ${ }^{\# \# \# ~} p<0.001$ vs. the vehicle PMCAO group) and the expression of P62 (Figure 5C, ${ }^{\#} p<0.05$ vs. the vehicle PMCAO group) were further downregulated in the AMCAO group. In contrast, after treatment with the autophagy inhibitor 3MA, the ratio of LC3II/LC3I was decreased (Figure 5B, $\&_{p}<0.05$ vs. the AMCAO group) and the protein level of P62 was increased (Figure 5C, ${ }^{\&} p<0.05$ vs. the AMCAO group). These results indicated that $3 \mathrm{MA}$ inhibited the autophagic flux (the increase of P62) in the 3MAMCAO group. In addition, pretreatment with ATV had a tendency to increase the pmTOR protein expression in the AMCAO group compared with that in the vehicle PMCAO group (\#\# $p<0.001$ ); however, 3MA reduced the phospho-mTOR expression in the 3MAMCAO group compared to that in the AMCAO group $\left({ }^{\&} \& p<0.01\right)$ (Figures 5A,D). In the vehicle PMCAO group, HIF-1 $\alpha$, induced by hypoxia (Wang et al., 2017) and dependent on both mTORC1 and mTORC2 (Masoud and Li, 2015), was increased compared to those in the SHAM controls and AMCAO group $\left({ }^{* *} p<0.01\right)$. In contrast, pretreatment with $3 \mathrm{MA}$ resulted in a significant increase of HIF- $1 \alpha$ in the 3MAMCAO group compared to the level in the AMCAO group $(\& \& \& p<0.001$, Figures 5E,F). An explanation for this may be that ATV mainly relieves P62 expression in the 


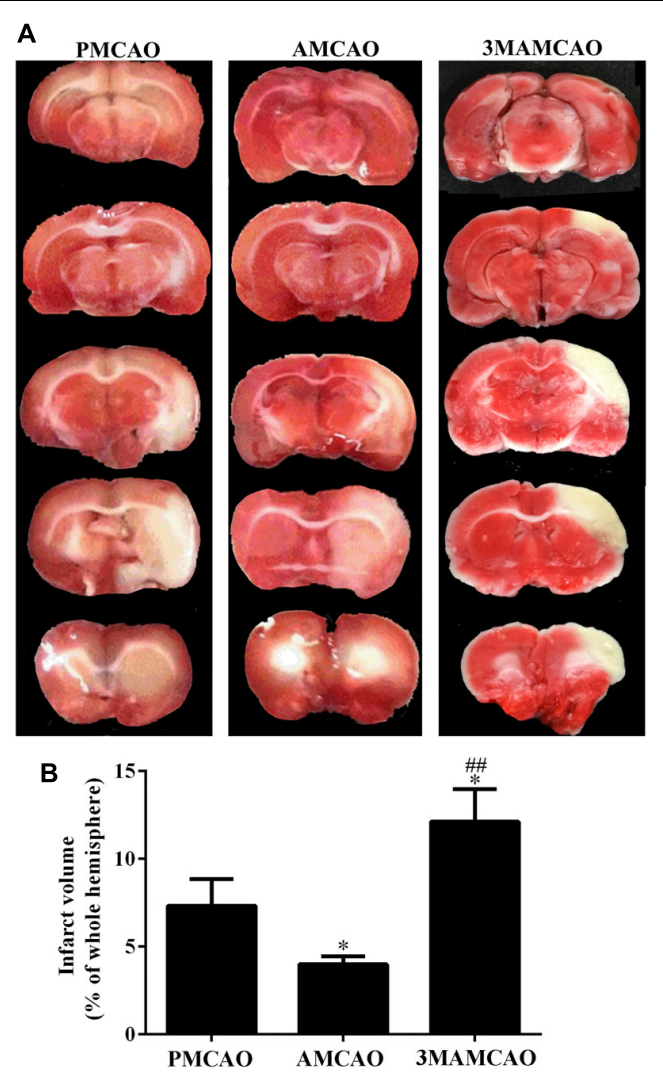

FIGURE 8 | TTC staining. (A) Infarct volume ratios in the brains of the PMCAO, AMCAO and 3MAMCAO animals at $24 \mathrm{~h}$ after MCAO surgery. The white areas represent the infarct. (B) The percentages of infarct volume in the brains. $N=4$, mean \pm SEM $^{*} p<0.05$ vs. PMCAO. ${ }^{\# \#} p<$ vs. AMCAO.

vehicle PMCAO group to inhibit hypoxia damage, whereas 3MA ablated the effect of ATV on the P62 expression that would have affected the attenuation in autophagy.

\section{Autophagy Inhibition Affected ER Stress in Rats With ATV Pretreatment and PMCAO}

Previous studies suggested that ischemia can activate ER stress (Drevinge et al., 2013). Herein, in the vehicle PMCAO group, the UPR was initiated, which is aimed at restoring the ER environment. In response to sustained ER stress, PERK activated ATF4 and phosphorylated eIF2 $\alpha$ to increase the expression of CHOP. CHOP and eIF2 $\alpha$ might provide an indicator of the cell condition and risk for apoptosis (Mantuano et al., 2011; Gharibani et al., 2013) (Figure 6A). In addition to PERK, the other two ER membrane-associated proteins that activate the UPR were ATF6 (Figure 6D) and IRE1, and the latter promotes the phosphorylation of JNK, P38, and NF- $\kappa$ B kinase (Figure 6B). However, pretreatment with ATV disturbed the activation of chaperones such as Grp78/Bip dissociation from IRE1- $\alpha$, PERK, and ATF6 during acute ER stress (Yin et al., 2017), as well as calnexin, Nrf2 and Ero1-L $\alpha$, which participate in oxidative and $\mathrm{Ca}^{2+}$-related stress to regulate ER stress (Giorgi et al., 2015) (Figure 6C), and downregulated the downstream activation of JNK, P38 and NF- $\mathrm{B}$ kinase (Figure 6B) to inhibit the subsequent apoptosis ( $\mathrm{Ma}$ et al., 2017). Furthermore, there was no significance in the change in Erol-L $\alpha$ between the vehicle PMCAO and AMCAO groups (Figure 6C). Indeed, $3 \mathrm{MA}$ prevents autophagy at an early stage of autophagosome formation. Pretreatment with 3MA and ATV in the 3MAMCAO group altered the ER stress response compared to that in the AMCAO group. 3MA treatment in AMCAO rats increased the expression of PERK and the downstream protein IRE1- $\alpha$, ATF4, CHOP, pNF- $\kappa$, and $\mathrm{pJNK}$, as well as activated the chaperones Grp78/Bip, calnexin, and Nrf2. It was not clear whether 3MA treatment for AMCAO further decreased the activation of eIF2 $\alpha$, pP38, Ero1-L $\alpha$, or ATF6. In summary, ER stress could be partially upregulated after autophagy inhibition and partially downregulated via other proteins after 3MA application in the 3MAMCAO group. To determine the effect of the partially changed proteins, the key apoptosis and anti-apoptosis proteins were detected.

\section{Autophagy Inhibition Enhanced ER Stress-Related Apoptosis and Brain Injury}

Having determined that ER stress activated caspase 3, we found that cleaved-caspase 3 was upregulated in the vehicle PMCAO group $\left({ }^{*} p<0.05\right.$ vs. the SHAM controls), but pretreatment with ATV significantly inhibited PMCAO-induced ER stress and the upregulation of cleaved-caspase $3(\# \#<0.001$ vs. the PMCAO group). However, pretreatment of the autophagy inhibitor $3 \mathrm{MA}$ in the $3 \mathrm{MAMCAO}$ rats significantly promoted cleaved-caspase 3, indicating that autophagic inhibition hindered the effect of ATV pretreatment in ischemic rats $\left({ }^{\&} \& p<0.01\right.$ vs. the AMCAO group, Figures 7A,B). On the other hand, pretreatment with ATV enhanced Bcl-2 in the vehicle PMCAO group ( ${ }^{*} p<0.01$ vs. the PMCAO group), while pretreatment with the autophagy inhibitor 3MA in the 3MAMCAO rats significantly reduced $\mathrm{Bcl}-2$ expression $(\& \& p<0.01$ vs. the AMCAO group, Figures 7A,C). Furthermore, the infarct volume ratio in the ATV-pretreated group was significantly decreased compared with that in the PMCAO group $\left({ }^{*} p<0.05\right)$. Furthermore, the infarct volume ratio in the 3MA-pretreated group was increased compared with that in the ATV-pretreated group ( ${ }^{\# \#} p<0.01$ ) (Figures 8A,B). These findings suggested that the possible inhibition of autophagy resulted in a reduction in the protective effect of ATV pretreatment.

\section{DISCUSSION}

Guidelines for the prevention of stroke and transient ischemic attack recommend statin therapy, which should be initiated for the secondary prevention of patients to reduce the frequency of first and recurrent ischemic stroke. Although Hong et al. found that pretreatment with a low dose of ATV could protect neurons in the ischemic penumbra and reduce the infarct volume in MCAO-reperfusion rats, which depends on vascular recanalization, according to the 


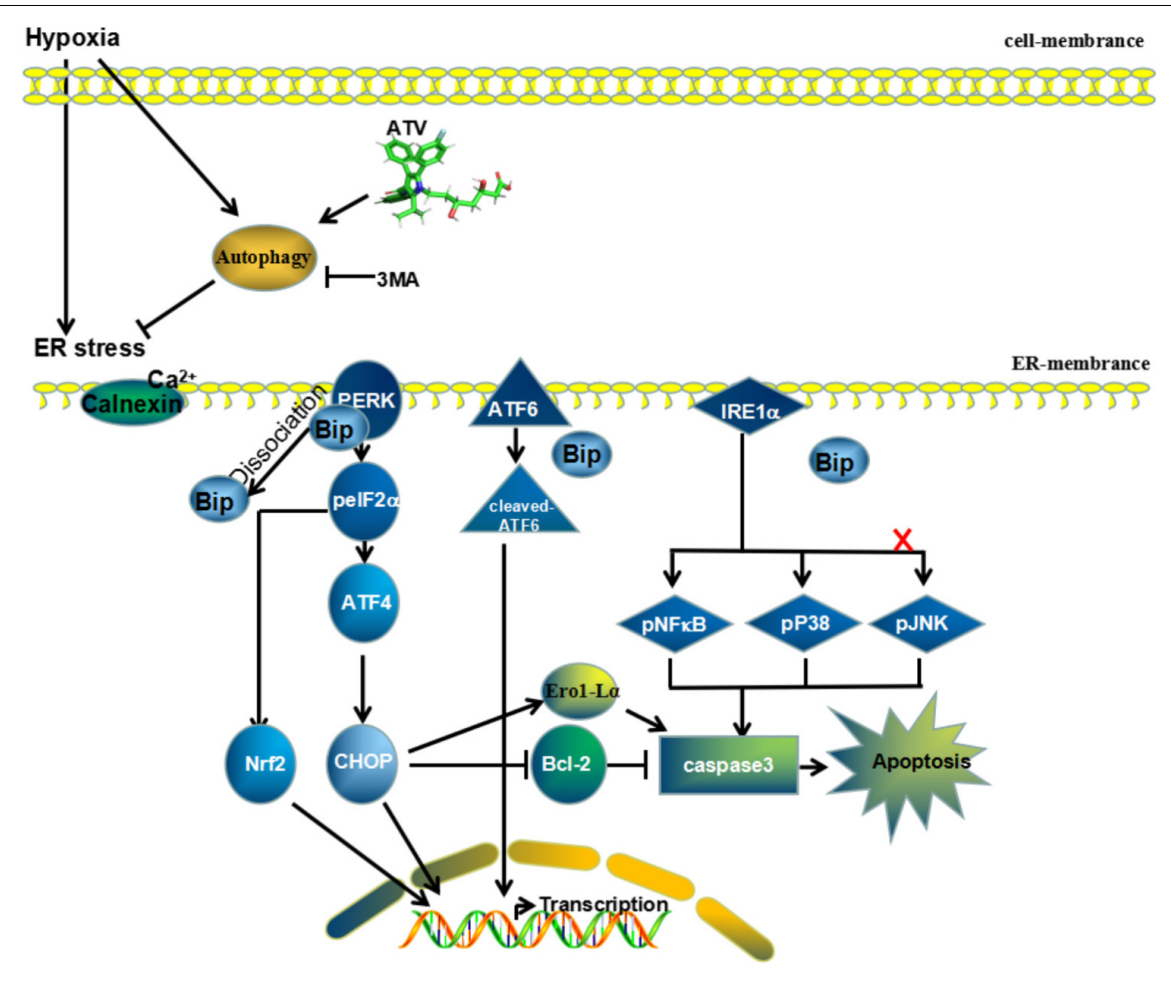

FIGURE 9 | Mechanism of ATV effects on MCAO injury. Potential neuroprotective mechanisms of ATV pretreatment for permanent middle artery occlusion through downregulation of the ER stress-related pathways PERK/elF2 $\alpha / A T F 4$ and IRE-1/NF-kB by enhancing autophagy flux. Compounds such as 3MA that inhibit autophagic activity will reduce the neuroprotective effect of ATV after brain ischemia.

survey results of the "Eleventh Five-Year Plan" key projects supported by National Science and Technology, the venous thrombolysis rate for cerebral infarction in China was only $1.3 \%$ (Tripathi et al., 2016) and approximately 10\% abroad. Indeed, without urgent reperfusion management after MCAO, neurons ultimately advanced toward apoptotic death. Therefore, for most patients with cerebral infarction who are unable to receive vascular recanalization, whether or not statins can decrease the infarct size and increase the survival rate of normal brain cells is the focus of further research. The mechanisms underlying the protective effect of statins need to be further explored, which may expand the indications for statin treatment.

In this study, we investigated the damage changes in different cells in the peri-infarct areas. From MRI, we tentatively observed the infarct volume on DWI and ADC values and blood flow reflected by $\mathrm{T}_{2}{ }^{*}$ in an MCAO rat brain (Liu et al., 2017). Vascular endotheliocytes were the first vulnerable cells compared with others following ischemia by toxic cytoplasmic calcium to affect the blood-brain barrier (BBB) integrity and neural cell viability (Posada-Duque et al., 2014). However, ATV treatment significantly limited the diffusion of water molecules and alleviated cytotoxic edema in this study. In addition, we found angiogenesis in the peri-infarct areas in the PMCAO rats, as well as increased numbers of astrocytes and microglia (Posada-Duque et al., 2014). In the MCAO rats that received ATV pretreatment, neurons and vascular endotheliocytes were promoted. In contrast, there was no significant difference in the number of microglia or astrocytes.

Under ultrastructural observation, damaged neurons were accompanied by autophagosomes and vacuolization of ER components. Recently, studies have reported that markedly increased autophagy following the upregulation of LC3 and downregulation of $\mathrm{P} 62$ in the peri-infarct areas following ischemia may reduce the infarct size, which was also validated in the vascular endotheliocytes and neurons in the AMCAO group. In comparison with the PMCAO control group, the number of vascular endothelial cells and neurons was higher after ATV pretreatment, and the ratio of P62-labeled cells/DAPI, P62 protein levels, the ratio of LC3-labeled neurons and LC3II/LC3I were decreased (Gao et al., 2012; Ma et al., 2017). Consistent with this phenomenon, 3MA pretreatment in the 3MAMCAO rats led to the stimulation of apoptosis and enlargement of the infarct, which were verified by the following results. LC3 as the main marker of autophagy flux provided a more accurate measurement of autophagic activity, and another marker, P62, specifically target ubiquitin-binding molecules for autophagic degradation and is inversely related to autophagic activity. When autophagy was initiated, LC3I was modified into the phosphatidylethanolamine-conjugated form LC3II, and LC3II was incorporated into autophagic vacuoles until degraded by lysosomes, whether P62 activation decided the downstream changes in autophagy (Yoshii and Mizushima, 2017). The autophagy processes in focal ischemia were disturbed by 3MA 
through the downregulation of LC3II and the upregulation of P62. Next, minimizing damage surrounding the ischemic areas requires the amelioration/prevention of cell apoptosis, which is the current vital therapeutic target in the treatment of acute ischemic stroke (Ma et al., 2017). In the current study, the hypoxia-related protein HIF1 $\alpha$ and the apoptotic protein cleaved-caspase 3 were attenuated, and the anti-apoptotic protein Bcl-2 was activated by ATV compared to that in the PMCAO group. Our study demonstrated that interference with autophagy might inhibit the effect of ATV pretreatment. Thus, the neuroprotective function of ATV depends upon autophagic activity, and the combined therapy that inhibits autophagic activity would inhibit the neuroprotective effect of ATV after brain ischemia.

To further discuss autophagy-induced apoptosis, we found in the PMCAO groups that autophagosomes were triggered by ER swelling in the ischemic area, and in turn, autophagy also affects ER stress. Cross talking with UPR/ER effector molecules, LC3 canonically triggers the degradation of organelles/molecules in the cytosol and potentiates the adaptive ability of the cell by clearing the load of misfolded proteins from the ER lumen (Rahman et al., 2017) to affect apoptosis (Talma et al., 2016). ER stress is initiated through the IRE-1, PERK, and ATF6 pathways in mammalian cells (Nakka et al., 2016; Rahman et al., 2017). IRE1 $\alpha$ from the ER might be involved in integrating ERS signaling with inflammatory-response signaling for activating both JNK and NF- $\mathrm{B}$ (Wu et al., 2014) to trigger cell apoptosis. In some ways, the permanent brain ischemia significantly enhanced the ER stress through PERK/eIF2 $\alpha /$ ATF4, ATF6 and IRE-1/NF$\kappa \mathrm{B} / \mathrm{JNK} / \mathrm{p} 38$ signaling. With ATV pretreatment, the molecular chaperone proteins were diminished, such as $\mathrm{CHOP}$ regulated by the mTOR pathway (Ma et al., 2018), Grp78 (Louessard et al., 2017), the calcium overloaded-related protein calnexin (Zhang et al., 2016b,a), and the oxidative system proteins Ero1L $\alpha$ (Capriotti and Murphy, 2016) and Nrf2 (Bocci and Valacchi, 2015; Zhang et al., 2017). However, the expression of pJNK and ATF6 was not significantly different between the AMCAO group and the vehicle PMCAO group. Cell apoptosis triggered by these proteins via MCAO stress could be inhibited by ATV pretreatment. The autophagy inhibitor, 3MA, could attenuate ATP generation, affect mTORC1 activation and lead to cell death in response to nutrition deprivation. It reduced the autophagy flux mainly via P62 upregulation. We supposed that autophagy might be the regulator of ER stress in statin-pretreated MCAO brains (Figure 9).

However, our study had several limitations. We only showed that the autophagy inhibitor 3MA potentially alters the neuroprotective effects of ATV in vivo; there was a lack of experiments to validate whether the autophagy activators benefit the protective effect of ATV. Future studies are needed to define

\section{REFERENCES}

Bin Abdulhak, A. A., Baddour, L. M., Erwin, P. J., Hoen, B., Chu, V. H., Mensah, G. A., et al. (2014). Global and regional burden of infective endocarditis, 19902010: a systematic review of the literature. Glob. Heart 9, 131-143. doi: 10.1016/ j.gheart.2014.01.002 the mechanism of autophagy activators for ATV therapy and the changes in glia in more animals.

These findings firmly indicated that the protective effects of ATV pretreatment were related to the reduction in autophagic activity and ER stress in vascular endothelial cells and neurons. In fact, the promotion of P62 significantly weakened the reduction in autophagic activity to diminish the ER stress in ATV pretreatment; in other words, our study provides a new view for ATV therapy, namely, that avoiding P62 hoarding or increasing autophagosome degradation might enhance the effect and expand the clinical indications of ATV in the treatment of acute ischemic stroke.

\section{AUTHOR CONTRIBUTIONS}

TZ completed the most draft writing. DL completed the most experiments. WY revised the draft. CS analyzed the MRI parts. JZ repeated the WB results. LS repeated the IF results. HM feed the rats. AX designed the whole experiments and draft, and submitted the manuscript to the Journal of Cellular Neuroscience.

\section{FUNDING}

This study was supported by the National Natural Science Foundation of China (81671167), Guangzhou Science and Technology Program key projects (2014Y2-00505), Guangzhou Science and Technology Program key projects (201508020004), Guangdong Medical Research Foundation (2015123184824609), and Science and Technology Planning Project of Guangdong Province, China (2017A020215049).

\section{ACKNOWLEDGMENTS}

The authors thank the American Journal Experts (Durham, North Carolina, United States) for improving our English.

\section{SUPPLEMENTARY MATERIAL}

The Supplementary Material for this article can be found online at: https://www.frontiersin.org/articles/10.3389/fnins. 2018.00405/full\#supplementary-material

DATA SHEET S1 | The whole raw bands of Western Blot exposed using Tanon 2500 Gel Imaging System, including the bands of CD34, VE-cadherin in Figure 2, pmTOR, LC3B, P62, and HIF1 $\alpha$ in Figure 5, ER stress pathway protein in Figure 6, and cleaved-caspase 3 and $\mathrm{Bcl}-2$ in Figure 7.

Bocci, V., and Valacchi, G. (2015). Nrf2 activation as target to implement therapeutic treatments. Front. Chem. 3:4. doi: 10.3389/fchem.2015. 00004

Cappellari, M., Bovi, P., Moretto, G., Zini, A., Nencini, P., Sessa, M., et al. (2013). The THRombolysis and STatins (THRaST) study. Neurology 80, 655-661. doi: 10.1212/WNL.0b013e318281cc83 
Cappellari, M., Bovi, P., Toni, D., Micheletti, N., Tomelleri, G., Carletti, M., et al. (2015). Cardioembolic stroke in the THRombolysis and STatins (THRaST) study. Int. J. Stroke 10:E22. doi: 10.1111/ijs.12355

Capriotti, T., and Murphy, T. (2016). Ischemic stroke. Home Healthc. Now 34, 259-266. doi: 10.1097/NHH.0000000000000387

Cui, L., Zhang, X., Yang, R., Wang, L., Liu, L., Li, M., et al. (2010). Neuroprotection of early and short-time applying atorvastatin in the acute phase of cerebral ischemia: down-regulated 12/15-LOX, p38MAPK and cPLA2 expression, ameliorated BBB permeability. Brain Res. 1325, 164-173. doi: 10.1016/j. brainres.2010.02.036

Drevinge, C., Karlsson, L. O., Stahlman, M., Larsson, T., Perman Sundelin, J., Grip, L., et al. (2013). Cholesteryl esters accumulate in the heart in a porcine model of ischemia and reperfusion. PLoS One 8:e61942. doi: 10.1371/journal. pone. 0061942

Gao, L., Jiang, T., Guo, J., Liu, Y., Cui, G., Gu, L., et al. (2012). Inhibition of autophagy contributes to ischemic postconditioning-induced neuroprotection against focal cerebral ischemia in rats. PLoS One 7:e46092. doi: 10.1371/journal. pone. 0046092

Garbuzova-Davis, S., Haller, E., Williams, S. N., Haim, E. D., Tajiri, N., HernandezOntiveros, D. G., et al. (2014). Compromised blood-brain barrier competence in remote brain areas in ischemic stroke rats at the chronic stage. J. Comp. Neurol. 522, 3120-3137. doi: 10.1002/cne.23582

Gharibani, P. M., Modi, J., Pan, C., Menzie, J., Ma, Z., Chen, P. C., et al. (2013). The mechanism of taurine protection against endoplasmic reticulum stress in an animal stroke model of cerebral artery occlusion and stroke-related conditions in primary neuronal cell culture. Adv. Exp. Med. Biol. 776, 241-258. doi: 10.1007/978-1-4614-6093-0_23

Giorgi, C., Bonora, M., Sorrentino, G., Missiroli, S., Poletti, F., Suski, J. M., et al. (2015). p53 at the endoplasmic reticulum regulates apoptosis in a $\mathrm{Ca}^{2+}$. dependent manner. Proc. Natl. Acad. Sci. U.S.A. 112, 1779-1784. doi: 10.1073/ pnas. 1410723112

Guo, D., Ma, J., Yan, L., Li, T., Li, Z., Han, X., et al. (2017). Downregulation of Lncrna MALAT1 attenuates neuronal cell death through suppressing beclin1-dependent autophagy by regulating mir-30a in cerebral ischemic stroke. Cell Physiol. Biochem. 43, 182-194. doi: 10.1159/0004 80337

Kubota, C., Torii, S., Hou, N., Saito, N., Yoshimoto, Y., Imai, H., et al. (2010). Constitutive reactive oxygen species generation from autophagosome/lysosome in neuronal oxidative toxicity. J. Biol. Chem. 285, 667-674. doi: 10.1074/jbc. M109.053058

Liu, Q., Wang, J. J., Yan, H. Y., Wang, Y. Q., Qin, C. W., and Li, M. L. (2017). Safety and pharmacodynamic mechanism of ropivacaine lumbar anesthesia in Cesarean section. J. Biol. Regul. Homeost. Agents 31, $71-76$.

Louessard, M., Bardou, I., Lemarchand, E., Thiebaut, A. M., Parcq, J., Leprince, J., et al. (2017). Activation of cell surface GRP78 decreases endoplasmic reticulum stress and neuronal death. Cell Death Differ. 24, 1518-1529. doi: 10.1038/cdd. 2017.35

Lu, D., Xu, A., Mai, H., Zhao, J., Zhang, C., Qi, R., et al. (2015). The synergistic effects of heat shock protein 70 and ginsenoside Rg1 against tert-butyl hydroperoxide damage model in vitro. Oxid. Med. Cell. Longev. 2015:437127. doi: $10.1155 / 2015 / 437127$

Ma, D., Feng, L., Deng, F., and Feng, J. C. (2017). Overview of experimental and clinical findings regarding the neuroprotective effects of cerebral ischemic postconditioning. Biomed Res. Int. 2017:6891645. doi: 10.1155/2017/ 6891645

Ma, L. L., Ma, X., Kong, F. J., Guo, J. J., Shi, H. T., Zhu, J. B., et al. (2018). Mammalian target of rapamycin inhibition attenuates myocardial ischaemiareperfusion injury in hypertrophic heart. J. Cell Mol. Med. 22, 1708-1719. doi: $10.1111 /$ jcmm. 13451

Mantuano, E., Henry, K., Yamauchi, T., Hiramatsu, N., Yamauchi, K., Orita, S., et al. (2011). The unfolded protein response is a major mechanism by which LRP1 regulates Schwann cell survival after injury. J. Neurosci. 31, 13376-13385. doi: 10.1523/JNEUROSCI.2850-11.2011

Masoud, G. N., and Li, W. (2015). HIF-1alpha pathway: role, regulation and intervention for cancer therapy. Acta Pharm. Sin. B 5, 378-389. doi: 10.1016/ j.apsb.2015.05.007
Nakka, V. P., Prakash-Babu, P., and Vemuganti, R. (2016). Crosstalk between endoplasmic reticulum stress, oxidative stress, and autophagy: potential therapeutic targets for acute CNS injuries. Mol. Neurobiol. 53, 532-544. doi: 10.1007/s12035-014-9029-6

Posada-Duque, R. A., Barreto, G. E., and Cardona-Gomez, G. P. (2014). Protection after stroke: cellular effectors of neurovascular unit integrity. Front. Cell. Neurosci. 8:231. doi: 10.3389/fncel.2014.00231

Rahman, S., Jan, A. T., Ayyagari, A., Kim, J., Kim, J., and Minakshi, R. (2017). Entanglement of UPRER in aging driven neurodegenerative diseases. Front. Aging Neurosci. 9:341. doi: 10.3389/fnagi.2017.00341

Rami, A., and Kogel, D. (2008). Apoptosis meets autophagy-like cell death in the ischemic penumbra: two sides of the same coin? Autophagy 4, $422-426$.

Talma, N., Kok, W. F., de Veij Mestdagh, C. F., Shanbhag, N. C., Bouma, H. R., and Henning, R. H. (2016). Neuroprotective hypothermia - Why keep your head cool during ischemia and reperfusion. Biochim. Biophys. Acta 1860, 2521-2528. doi: 10.1016/j.bbagen.2016.07.024

Tekle, W. G., Chaudhry, S. A., Fatima, Z., Ahmed, M., Khalil, S., Hassan, A. E., et al. (2012). Intravenous thrombolysis in expanded time window (3-4.5 hours) in general practice with concurrent availability of endovascular treatment. J. Vasc. Interv. Neurol. 5, 22-26.

Tripathi, M., Zhang, C. W., Singh, B. K., Sinha, R. A., Moe, K. T., DeSilva, D. A., et al. (2016). Hyperhomocysteinemia causes ER stress and impaired autophagy that is reversed by Vitamin B supplementation. Cell Death Dis. 7:e2513. doi: 10.1038/cddis.2016.374

Wang, L., Jin, Z., Wang, J., Chen, S., Dai, L., Lin, D., et al. (2017). Detrimental effect of Hypoxia-inducible factor-1alpha-induced autophagy on multiterritory perforator flap survival in rats. Sci. Rep. 7:11791. doi: 10.1038/s41598-01712034-x

Wang, L., Zhang, X., Liu, L., Yang, R., Cui, L., and Li, M. (2010). Atorvastatin protects rat brains against permanent focal ischemia and downregulates HMGB1, HMGB1 receptors (RAGE and TLR4), NFkappaB expression. Neurosci. Lett. 471, 152-156. doi: 10.1016/j.neulet.2010. 01.030

Wang, P., Shao, B. Z., Deng, Z., Chen, S., Yue, Z., and Miao, C. Y. (2018). Autophagy in ischemic stroke. Prog. Neurobiol. 163-164, 98-117. doi: 10.1016/ j.pneurobio.2018.01.001

Wu, Q., Wang, Q., Guo, Z., Shang, Y., Zhang, L., and Gong, S. (2014). Nuclear factor-kappaB as a link between endoplasmic reticulum stress and inflammation during cardiomyocyte hypoxia/reoxygenation. Cell Biol. Int. 38, 881-887. doi: 10.1002/cbin. 10272

Yang, J. W., and Hu, Z. P. (2015). Neuroprotective effects of atorvastatin against cerebral ischemia/reperfusion injury through the inhibition of endoplasmic reticulum stress. Neural Regen. Res. 10, 1239-1244. doi: 10.4103/1673-5374. 162755

Yin, H., Zhao, L., Jiang, X., Li, S., Huo, H., and Chen, H. (2017). DEV induce autophagy via the endoplasmic reticulum stress related unfolded protein response. PLoS One 12:e189704. doi: 10.1371/journal.pone. 0189704

Yoshii, S. R., and Mizushima, N. (2017). Monitoring and measuring autophagy. Int. J. Mol. Sci. 18:E1865. doi: 10.3390/ijms18091865

Zhang, R., Xu, M., Wang, Y., Xie, F., Zhang, G., and Qin, X. (2017). Nrf2a promising therapeutic target for defensing against oxidative stress in stroke. Mol. Neurobiol. 54, 6006-6017. doi: 10.1007/s12035-0160111-0

Zhang, X., Zhang, J., Li, R., Cheng, G., and Chen, Q. (2016a). Understanding the reasons behind the low utilization of thrombolysis in hyperacute ischemic stroke. Zhonghua Yi Xue Za Zhi 96, 863-867. doi: 10.3760/cma.j.issn.0376-2491. 2016.11.008

Zhang, Y., Wang, T., Yang, K., Xu, J., Ren, L., Li, W., et al. (2016b). Cerebral microvascular endothelial cell apoptosis after ischemia: role of enolase-phosphatase 1 activation and aci-reductone dioxygenase 1 translocation. Front. Mol. Neurosci. 9:79. doi: 10.3389/fnmol.2016. 00079

Zhang, Y., Xing, S., Zhang, J., Li, J., Li, C., Pei, Z., et al. (2011). Reduction of beta-amyloid deposits by gamma-secretase inhibitor is associated with the attenuation of secondary damage in the ipsilateral thalamus and sensory 
functional improvement after focal cortical infarction in hypertensive rats. J. Cereb. Blood Flow Metab. 31, 572-579. doi: 10.1038/jcbfm.2010.127

Zhao, Y., Huang, G., Chen, S., Gou, Y., Dong, Z., and Zhang, X. (2016). Homocysteine aggravates cortical neural cell injury through neuronal autophagy overactivation following rat cerebral ischemiareperfusion. Int. J. Mol. Sci. 17:1196. doi: 10.3390/ijms1708 1196

Zhu, H., Zhang, Y., Shi, Z., Lu, D., Li, T., Ding, Y., et al. (2016). The neuroprotection of liraglutide against ischaemia-induced apoptosis through the activation of the PI3K/AKT and MAPK pathways. Sci. Rep. 6:26859. doi: 10.1038/srep 26859
Conflict of Interest Statement: The authors declare that the research was conducted in the absence of any commercial or financial relationships that could be construed as a potential conflict of interest.

Copyright $\odot 2018$ Zhang, Lu, Yang, Shi, Zang, Shen, Mai and Xu. This is an openaccess article distributed under the terms of the Creative Commons Attribution License (CC BY). The use, distribution or reproduction in other forums is permitted, provided the original author(s) and the copyright owner are credited and that the original publication in this journal is cited, in accordance with accepted academic practice. No use, distribution or reproduction is permitted which does not comply with these terms. 